\title{
Vitiligo-Like Patches due to Epidermal Interface Changes in Frontal Fibrosing Alopecia: Further Evidence of Non-Follicular Involvement
}

\author{
Coelho TOA*, Romiti R, Valente NS and Donati A
}

University of Sao Paulo, Brazil

\begin{abstract}
Frontal Fibrosing Alopecia (FFA) is a primary cicatricial alopecia that presents with alopecia over the frontal hairline. Skin involvement in FFA was first acknowledged in 2012 when the association between FFA and Lichen Planus (LP) pigmentosus was described. Here we present a Caucasian FFA female patient with vitiligo-like patches over the front and neck whose biopsy showed epidermal interface changes. This is the first time achromic lesions due to interface dermatitis are associated to FFA, adding evidence for the extra-follicular epithelial involvement of the disease. True vitiligo, as well as subacute lupus erythematosus, are differential diagnoses that must be excluded.
\end{abstract}

\section{Introduction}

Frontal Fibrosing Alopecia (FFA) is a primary cicatricial alopecia that presents with typical band-like frontal alopecia, usually in postmenopausal women $[1,2]$. Frequent skin involvement in FFA was first acknowledged in 2012 when more than $50 \%$ of African FFA patients were shown to have hyperchromic macules on sunexposed areas and Lichen Planus (LP) pigmentosus was confirmed histologically showing lichenoid epidermal changes [1]. Here we present a Caucasian FFA patient who developed achromic macules over the front and neck, whose biopsy showed epidermal interface changes similar to LP pigmentosus.

\section{Case Report}

A 66-year-old Caucasian woman presented with symmetric bandlike alopecia at the frontal hairline as well as complete eyebrow loss for over 10 years. A biopsy of the scalp evidenced typical features of FFA, such as perifollicular lymphocytic infiltrate and fibrosis, and treatment with $400 \mathrm{mg}$ hydroxichloroquine and $5 \mathrm{mg}$ finasteride orally every day was initiated. One year later, achromic patches developed over sunexposed areas of her face and neck with no previous sign of skin inflammation. (Figure 1) She had systemic hypertension, type II diabetes mellitus and dyslipidemia for which she took atenolol, enalapril, metformin, ezetimibe and simvastatin. She denied a personal or family history of vitiligo and no familiar cases of autoimmune disorders were reported. Laboratory tests showed negative thyroid antibodies and Antinuclear Antibodies (ANA). Skin biopsy of an achromic macule of the neck showed epidermal atrophy and rectification with a reduced melanocyte count, fibrosis of the dermal papilla and pigmentary incontinence (Figure 2). Periodic acid shiff stain did not evidence any relevant basal membrane thickening. Direct Immunofluorescence (DIF) showed IgM granulous and continuous deposits over the basal membrane zone.

\section{Discussion}

FFA is a clinically distinctive follicular disorder of unknown origin. Histological similarity with Lichen Planopilaris (LPP) as well as increased association of FFA with vulvar and oral LP suggest it is part of the LP spectrum [3].

The coexistence of true vitiligo and FFA has been reported by Miteva et al. as an example of a common genetic background for two different autoimmune disorders [2]. In our case, histopathology excluded the diagnosis of vitiligo and showed interface changes associated with pigment loss. Importantly, ANA and anti-Ro were negative and there were no histopathologic findings suggestive of lupus erythematosus [4]. Furthermore, DIF pattern was compatible with LP involvement.

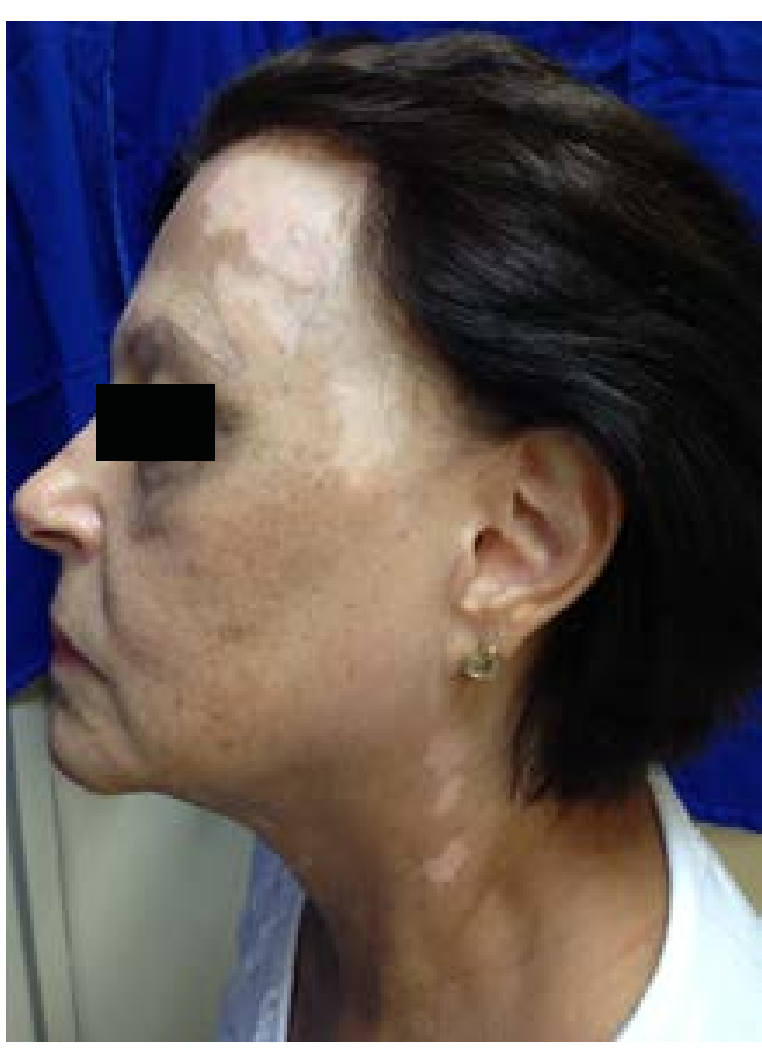

Figure 1: Clinical aspect showing symmetric band like alopecia of the frontal hairline, eyebrow loss and achromic macules over the front and neck.

*Corresponding author: Thaíssa OA Coelho, Trichology Fellow, University of Sao Paulo, Rua Padre Marinho, 49, room 802, Belo Horizonte, Sao Paulo, Brazil- 30140040, Tel: +55 (31) 30553564; E-mail:thaissadermato@gmail.com

Received November 14, 2014; Accepted November 22, 2014; Published November 25, 2014

Citation: Coelho TOA, Romiti R, Valente NS, Donati A (2014) Vitiligo-Like Patches due to Epidermal Interface Changes in Frontal Fibrosing Alopecia: Further Evidence of Non-Follicular Involvement. Pigmentary Disorders 2:153. doi:10.4172/23760427.1000153

Copyright: (c) 2014 Coelho TOA et al. This is an open-access article distributed under the terms of the Creative Commons Attribution License, which permits unrestricted use, distribution, and reproduction in any medium, provided the original author and source are credited. 


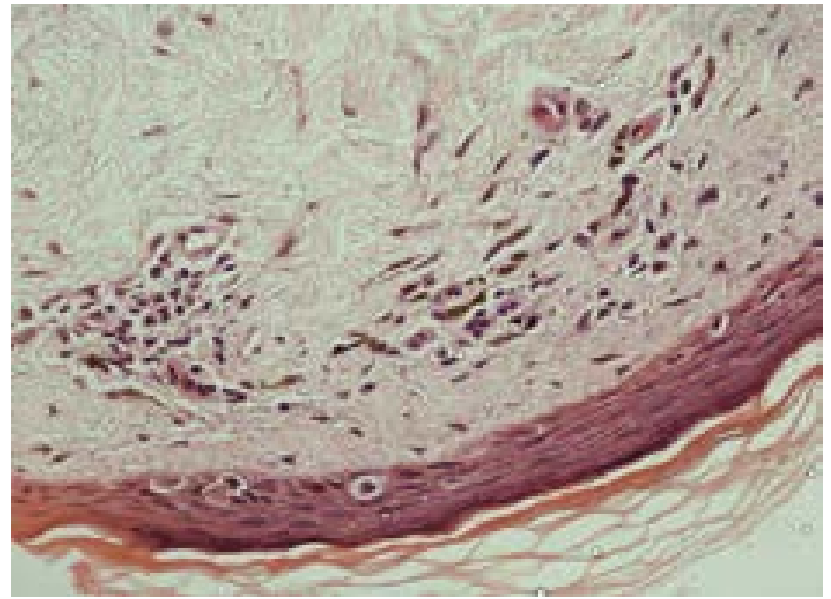

Figure 2: Skin biopsy of the achromic macule showing epidermal atrophy and pigmentary incontinence.

Dyschromic LP actinicus has been described and affects preferentially the posterior neck and dorsa of hands with achromic papules and small horny plugs, differently from our patient's presentation. Involvement of the face and neck is seen most often with the melasma-like variant of LP actinicus, but it is possible that the same lichenoid aggression leads to achromic lesions instead of hyperchromic ones, such as observed in vitiligoid subacute lupus erythematosus (SALE) $[5,6]$.

This case corroborates previous findings of cutaneous interface changes associated with FFA, but it is the first time achromic lesions related to interface dermatitis are reported in the setting of FFA. True vitiligo, as well as SALE, must be excluded by histology. Evidence of non-follicular epithelial involvement in FFA is increasing and highlights an important difference to classic LPP, raising the possibility of different pathogenic pathways.

\section{References}

1. Dlova NC (2013) Frontal fibrosing alopecia and lichen planus pigmentosus: is there a link? Br J Dermatol 168: 439-442.

2. Miteva M, Aber C, Torres F, Tosti A (2011) Frontal fibrosing alopecia occurring on scalp vitiligo: report of four cases. Br J Dermatol 165: 445-447.

3. Chew A, Stefanato CM, Savarese I, Neill SM, Fenton DA, et al. (2014) Clinical patterns of lichen planopilaris in patients with vulval lichen planus. $\mathrm{Br} \mathrm{J}$ Dermatol 170: 218-220.

4. Franca AF, de Souza EM (2010) Histopathology and immunohistochemistry of depigmented lesions in lupus erythematosus. J Cutan Pathol 37: 559-564.

5. Kim GH, Mikkilineni R (2007) Lichen planus actinicus. Dermatol Online J 13 13.

6. Meads SB, Kunishige J, Ramos-Caro FA, Hassanein AM (2003) Lichen planus actinicus. Cutis 72: 377-81. 